\title{
Zur idiomatischen Komponente auffälliger kausativer Konstruktionen im Deutschen
}

\author{
Martine Dalmas (Sorbonne) und Laurent Gautier (Bourgogne Franche-Comté)
}

\begin{abstract}
This paper aims to analyze German caused-motion constructions such as jn aus dem Bett klingeln from both a phraseological and constructional perspective. Section 1 shows how the traditional definition of phraseologisms is nowadays challenged by new developments in construction grammar, especially within Goldberg's framework. Section 2 focuses on the various possibilities existing in German to express causativity and proposes a continuum from mere morphological signs to the constructions under consideration here. Section 3 exemplifies the cognitive inscription of these causative constructions through the underlying frame semantic level. In section 4 we finally argue that several aspects of these structures, such as lexical constraint, figuration, and productivity suggest that some kinds of constructions should be integrated, at least peripherally, into the phraseological system of the given language.
\end{abstract}

\section{$1 \quad$ Ausgangspunkt und Problemstellung}

Ziel des Aufsatzes ist es, bestimmte caused-motion-Konstruktionen auf ihre Idiomatizität hin zu prüfen und ihre Anbindung an die Phraseologie kritisch zu hinterfragen. Vor diesem Hintergrund lässt sich die Untersuchung theoretisch-methodologisch zwischen drei Forschungstraditionen verorten.

Dem Schwerpunkt dieses thematischen Heftes entsprechend, wird an erster Stelle die Konstruktionsgrammatik in ihrer Goldbergschen Prägung (Goldberg 1995, 2006) bemüht: Es soll die These exemplifiziert werden, nach der hinter den ausdrucksseitig sehr unterschiedlichen Möglichkeiten, im Deutschen Kausativität auszudrücken, eine gemeinsame abstrakte Struktur vorliegt, die konstruktionsgrammatisch und zum Teil idiomatisch-phraseologisch gedeutet werden kann.

Diese Arbeit reiht sich auch in gewisser Hinsicht in die ,traditionelle' Forschung zur Idiomatizität (Burger 1973; Burger/Buhofer/Sialm 1982; Fleischer 1997; Burger 2010; Burger/Dobrovol'skij/Kühn/Norrick 2007) ein, mit ihren drei graduell aufgefassten Definitionskriterien vorgeformter Strukturen: 
1. Polylexikalität: Als Phraseme werden Einheiten anerkannt, die aus mehr als einem (typographischen) Wort bestehen (so genannte Mehrwortlexeme), lexikalisch gesehen aber wie ein einfaches Lexem funktionieren, wie dies zum Beispiel durch die Gegenüberstellung des polylexikalischen Funktionsverbgefüges zum Ausdruck kommen mit dem Verb ausdrücken ersichtlich wird (cf. Kamber 2008);

2. Figuriertheit: Phraseme haben eine Gesamtbedeutung, die sich nicht automatisch aus der Summe der Bedeutungen ihrer Formative ergibt (Nicht-Kompositionalität); im Phrasem mit dem Feuer spielen genügt die Kenntnis der beiden Elemente Feuer und spielen nicht, um die Bedeutung ein Risiko eingehen zu rekonstruieren (cf. Dobrovol'skij/Piirainen 2009).

Diese Darstellung folgt der germanisch-slawischen Phraseologieforschung, die seit den 1980er Jahren mit diesen drei Kriterien operiert, auch wenn sie immer wieder hinterfragt bzw. relativiert wurden. In seinem Überblicksaufsatz, wo diese Tradition kaum wahrgenommen wird, schlägt Gries (2008) sechs Parameter zur Identifizierung von Phrasemen vor, die er auch als graduell einstuft. Mit der Diskussion des Parameters, lexikalisch gefüllt oder nicht` stellt er auch die Frage, die im Mittelpunkt dieses Beitrages steht, und zwar: Inwiefern können Argumentstrukturen in die Phraseologie Eingang finden?

Über die Phraseologie hinaus knüpft diese Studie drittens an die auch in Grammatiken seit langem usuell gewordenen Ansatz zur Beschreibung von (kausativen) Funktionsverbgefügen an (Helbig 1979; van Pottelberge 2001; Kamber 2008). Da die formelle - sprich: syntaktische Ebene alleine nicht ausreicht, um die Produktivität der hier behandelten Strukturen zu erklären, wird dieser Ansatz mit der eher angelsächsischen Tradition der Forschung zu formulaic language (Wray 2002, 2008; Granger/Meunier 2008) angereichert, welche die kognitive Seite in den Mittelpunkt rückt und die betroffenen Einheiten prozedural untersucht, sowohl bei der Produktion - wo diese mehr oder weniger abstrakt gespeicherten Routinen als Formulierungshilfen fungieren - als auch bei der Rezeption.

Den Untersuchungsgegenstand bildet ein besonderer Typ kausativer Konstruktionen ${ }^{1}$ wie in (1) und (2):

(1) Der Briefträger hat mich aus dem Bett geklingelt.

(2) Sie sang das Kind in den Schlaf.

Solche Äußerungen weisen nämlich eine auffällige Parallele mit caused-motion constructions, wie sie Goldberg (1995) fürs Englische postuliert und die inzwischen intensiv erforscht wurden (cf. Dixon 2000; Gries/Stefanowitsch 2004; Hampe 2011). Ihnen liegt ein ,normalerweise“ nicht transitives Verb zu Grunde, das hier transitiv und in Verbindung mit einer, Ortsangabe' verwendet wird, wobei diese Präpositionalphrase einen von der durch das Hauptverb ausgedrückten Handlung resultierenden Prozess im generischen Sinne des Wortes bezeichnet. Diese Einheiten scheinen uns fürs Deutsche unter einem gewissen Forschungsdefizit zu leiden, insbesondere im Vergleich zu resultativen Strukturen stricto sensu wie in (3), die ausführlich beschrieben wurden (cf. u. a. Müller 2002, Boas 2003, Dalmas 2006):

\footnotetext{
${ }^{1}$ An dieser Stelle wird der Begriff Konstruktion (noch) a-theoretisch verwendet, unter anderem um die systematische Wiederholung von Struktur zu vermeiden. Ziel des Aufsatzes ist es gerade, ihre theoretische Relevanz zu zeigen, um sie letzten Endes im Kontext der Goldbergschen Konstruktionsgrammatik zu interpretieren.
} 
(3) Essen Sie sich wieder gesund!

Dieses Forschungsdefizit mag wohl an ihrer gleichzeitig höheren Komplexität und niedrigeren Produktivität liegen. Sie stellen nämlich den Linguisten vor viele unbeantwortete Fragen: Wie lässt sich die Transitivierung der zu Grunde liegenden Verben erklären? Wie werden diese Strukturen, die an sich nicht völlig lexikalisiert sind, produziert und dekodiert, d. h. mit welchen semantischen Verbklassen sind sie möglich? In welchem Zusammenhang stehen sie sowohl mit den anderen Mitteln zum Ausdruck der Kausativität als auch mit den anderen Komponenten des constructicon deutscher Sprecher? Auf diese Fragen zu antworten macht es notwendig, auch ihren Stereotypisierungsgrad in Betracht zu ziehen.

Aus diesem Grund werden im Folgenden drei Arbeitshypothesen aufgestellt:

1. sie sind Teil eines syntaktisch-semantischen Konstruktions-Kontinuums und können nur vor diesem Gesamthintergrund analysiert werden;

2. ihre metaphorische Semantik beruht auf einem allgemeinen abstrakten Schema, das bei Muttersprachlern internalisiert wird und deren Rezeption und Produktion gewährleistet;

3. es sind augenfällige Berührungspunkte zwischen Phraseologie und Konstruktionsgrammatik und so stellt sich die Frage nach dem Stellenwert solcher abstrakten Prädikat-Argument-Ketten im Sprachsystem.

Unsere Argumentation beginnt mit einem kurzen Überblick zur Charakterisierung kausativer Verben und Konstruktionen im Deutschen, und zwar mit dem Ziel, die hier fokussierten Strukturen in den gesamten Zusammenhang der Kausativität einzubeziehen (Abschnitt 2). Von dieser Darstellung ausgehend werden dann die in Frage kommenden Einheiten kognitiv beleuchtet, wobei ein frame-semantisches Instrumentarium herangezogen wird (Abschnitt 3). In einem letzten Schritt wird abschließend die Frage nach ihrer phraseologischen Relevanz diskutiert (Abschnitt 4).

\section{Kausative Verben und kausative Konstruktionen: semantisch-syntaktische Charakterisierung}

Kausative Konstruktionen lassen sich in eine Reihe von Formen und Strukturen einordnen, die als Kontinuum betrachtet werden kann. An einem Ende finden wir lexikalische Formen (Lexeme oder feste Fügungen), am anderen Ende sind Konstruktionen anzusiedeln, deren lexikalische Füllung zwar relativ frei zu sein scheint, jedoch in Wirklichkeit gewissen Einschränkungen unterliegt.

- $\quad$ Es sind augenfällige Berührungspunkte zwischen Phraseologie und Konstruktionsgrammatik und so stellt sich die Frage nach dem Stellenwert solcher abstrakten Prädikat-Argument-Ketten im Sprachsystem.

- $\quad$ Das Deutsche verfügt bekanntlich nur über wenige minimale Oppositionssysteme, bei denen ein Verb eine kausative Entsprechung hat; Beispiele hierfür sind fallen > fällen; trinken > tränken; springen > sprengen. Neben der Tatsache, dass die kausativen Verben hier semantisch spezifizierter sind als ihre nicht kausative Variante, unterscheiden 
sich diese Verben vor allem durch ihre Argumentstruktur bzw. dadurch, dass sie jeweils eine andere Verteilung von syntaktischen Funktionen und semantischen Rollen aufweisen: Was bei dem nicht-kausativen Verb Subjekt (und Agens) ist, ist bei dem kausativen Verb Objekt (und Patiens):

(4) Landwirt fällte einen Baum - dieser fiel auf die Autobahn. ${ }^{2}$

Ähnlich verhält es sich bei der wohlbekannten Opposition zwischen Zustands- und Bewegungsverben: liegen > legen; sitzen > setzen; stehen > stellen usw. Hier wechselt der ,Zustandsträger' (experiencer) in die Rolle des Patiens. Außerdem enthält die kausative Struktur ein drittes Argument, das die direktionale Komponente des Syntagmas enthält und syntaktisch obligatorisch ist:

(5) Ein Kind liegt auf dem Bett und liest in einem Buch.

(6) Kari legt das Kind auf das Bett und macht Feuer.

Solche morphosyntaktischen Konsequenzen dürften - mitsamt ihrer rein morphologischen Merkmale - auch der Grund sein, warum diese Verben traditionell in Grammatiken gut etabliert sind.

- $\quad$ Es sind außerdem diese (Bewegungs-)Verben ${ }^{3}$, die als sog. Funktionsverben fungieren können und zum Ausdruck der Kausativität in Funktionsverbgefügen (FVG) dienen (z. B.: etwas in Bewegung/in Kraft/in Betrieb setzen; etwas zur Verfügung stellen). Es können aber auch andere Verben diese Funktion übernehmen: etwas in Erwägung ziehen; jdn. in die Irre führen; etwas zur Bearbeitung bringen ${ }^{4}$.

Interessant ist in allen diesen Fällen die direktionale Präpositionsphrase als fester Bestandteil des FVG - wobei die Direktivität durch die Präposition und/oder den Kasus markiert wird. Aufgrund ihrer relativen Festigkeit und ihrer reihenbildenden Potenz sind solche Gefüge in der deutschen Grammatikographie in den Bereich der Phraseologie gerückt (cf. van Pottelberge 2007).

- $\quad$ Neben diesen beiden Ausdrucksmöglichkeiten (kausatives Verb und FVG) gibt es auch Fälle, die weniger etabliert, lexikalisch weniger fest und deshalb auch weniger lexikalisiert sind, so zum Beispiel:

(7) Wie lange darf ich mein Kind in den Schlaf stillen?

Oder folgender Fall, mit einem Verb, das keine Spuren von Bewegung aufweist:

(8) Sie hat ihren Freund aus dem Bett geklingelt.

In solchen Fällen reicht eine morpho-syntaktische Beschreibung nicht mehr aus, denn die Konstruktion lässt sich nicht mehr vom Verb aus beschreiben (das Verb ist nicht immer transitiv und drückt keine zielgerichtete Bewegung aus). Hier ist es notwendig, die Beziehung FormBedeutung mit zu berücksichtigen, die nicht mehr isomorph ist und nicht mehr auf der WortEbene allein interpretiert werden kann. Solche Fügungen sind weitgehend mit englischen Konstruktionen vergleichbar wie etwa:

\footnotetext{
2 Wochenblatt, 16.11.2004.

3 ,Bewegung' bezeichnet hier eine zielorientierte Bewegung.

${ }^{4}$ Mehrere ausführliche Studien liegen zu diesen Aspekten vor, von Heringer (1968) bis Kamber (2006, 2008).
}

ISSN 1615-3014 
(9) They laughed the poor guy into his car/out of the auditorium/off the stage.

Auch wenn sie im Englischen öfter vorkommen, ist ihre Bildung im Deutschen auch mit unterschiedlichen Verben möglich, wie wir es weiter unten zeigen werden.

Die kausativen Formen, die wir hier erwähnt haben, ordnen sich in ein Kontinuum ein, das wie folgt gegliedert und exemplarisch illustriert werden kann. Ab Stufe $4 \mathrm{~b}$ sind die fett markierten Merkmale diejenigen, durch die sich die Syntax des einfachen Verbs von seinem Gebrauch in der Konstruktion unterscheidet und die den metaphorischen Prozess in Gang setzen:

Stufe 1: kausative Variante eines nicht-kausativen Verbs

z. B. ,Er hat den Baum gefällt.‘

Stufe 2: $\quad$ FVG mit kausativem Funktionsverb

z. B. ,Wir stellen Ihnen einen sicheren Platz zur Verfügung. ‘

Stufe 3: tr. Verb; Ortsveränderung: +; Akkusativergänzung: +, Direktivergänzung: +

z. B. ,Er hat das Geld auf den Tisch gelegt.“

Stufe 4a: intr./tr. Verb; Ortsveränderung: +; Akkusativergänzung: +/-;

Direktivergänzung: $+/-$

z. B. ,Er hat das Auto in den Graben gefahren.

Metaphorischer Gebrauch:

Stufe 4b: tr. Verb; Ortsveränderung: - ; Akkusativergänzung: + ; Direktivergänzung: + z. B. Er hat das Kind in den Schlaf gewiegt.

Stufe 5a: tr. Verb ${ }^{5}$; Ortsveränderung: - ; Akkusativergänzung: +;

Direktivergänzung: +

z. B. Er hat das Kind in den Schlaf gesungen

Stufe 5b: intr. Verb; Ortsveränderung: - ; Reflexivpronomen Akk +;

Direktivergänzung: +

z. B. Sie hat sich in die Herzen der Gäste getanzt. ${ }^{6}$

Stufe 5c: intr. Verb; Ortsveränderung: + ; Akkusativergänzung: +;

Direktivergänzung: +

z. B. Sie hat ihren Freund aus dem Bett geklingelt.

Die syntaktische Struktur auf Stufe 3 kann als die prototypische betrachtet werden, die dann bei den nächsten Stufen vorhanden bleibt. Besonders interessant werden die Konstruktionen ab Stufe 5, wenn das Verb in Verbindung mit einer Direktivergänzung sein Valenzschema ändert. Ab hier verändert sich nämlich fast die ganze syntaktische Umgebung des Verbs: Ein transitives Verb bekommt eine semantisch andere Akkusativergänzung oder ein Reflexivpronomen besetzt die Stelle einer Akkusativergänzung bzw. ein intransitives Verb wird von einer Akkusativergänzung begleitet. Da das Verb nicht unbedingt eine Bewegung, geschweige denn eine Orts-

\footnotetext{
5 In solchen Fällen bleibt das Verb zwar transitiv, aber es ändert seine semantische Valenz: Die Akkusativergänzung bezeichnet das Patiens, das von der verursachten Bewegung betroffen ist.

6 Solche Strukturen werden auch als ,reflexive-motion constructions“ bezeichnet (cf. Perek/Hilpert 2014). Die Bewegung wird nicht von einer externen Entität verursacht, sondern vom Agens selbst.
} 
veränderung ausdrücken muss, um eine solche Konstruktion zuzulassen, müssen wir die semantische Komponente, Ortsveränderung' vom Verb trennen, um sie der Gesamtkonstruktion zuzuschreiben. Diese Konstruktionen werden hier zwar als Abwandlungen ein- und derselben Struktur betrachtet; sie sind aber keineswegs vollsynonymisch austauschbar, insofern als sie pragma-semantisch gesehen - unterschiedliche mehr oder weniger konkrete bzw. abstrakte Sachverhalte enkodieren.

Im nächsten Teil gehen wir auf die Interpretation solcher Konstruktionen ${ }^{7}$ ein, die - bei aller scheinbaren lexikalischen Freiheit oder Unspezifiziertheit - gewisse Regeln befolgen und aufgrund bestimmter Wissensaspekte ohne großen kognitiven Aufwand interpretierbar sind.

\section{Kognitive Ebene}

Die Stabilität der postulierten abstrakten semantisch-syntaktischen Struktur kann unseres Erachtens mit Rückgriff auf die kognitive Ebene und insbesondere auf frame-semantische Modelle aufgedeckt werden. Es ist hier nicht der Ort, die einzelnen Etappen zu kommentieren, welche von der Frame-Semantik zur Konstruktionsgrammatik geführt haben bzw. führen: Sie sind schon reichlich diskutiert worden (cf. u. a. m. Nemoto 1998; Boas 2008; Fried 2009; Ziem 2014) und Goldberg selbst (1995: 25-31) baut ihr Modell z. T. auf den Ergebnissen der Fillmore'schen Bedeutungsbeschreibung von Verben auf. Bevor wir diesen Übergang von der Frame-Semantik zur Konstruktionsgrammatik auf die hier untersuchten Strukturen anzuwenden versuchen, sei das Analyseprinzip kurz an der so genannten ditransitiven Konstruktion veranschaulicht.

Die drei Gruppen von Belegen (10), (11) und (12) können alle auf ein- und dieselbe Konstruktion reduziert werden:

(10) a. Hast du den Pflanzen schon Dünger gegeben? ${ }^{8}$

b. Die Bundesrepublik schenkt Berlin ein Museum für die deutsche Geschichte.

c. Er händigte mir Empfehlungsschreiben meiner Freunde in Deutschland aus.

(11) a. Mama hatte mir das Nikolauspäckchen per Post nach München geschickt.

b. Sie übermittelten mir per E-Mail im März 2015 eine Anfrage des Urlaubers Herrn Kurt Müller.

c. Sie faxten mir freundlicherweise eine Anschrift, und ich bereitete mich darauf vor, wieder von vorn anzufangen.

(12) a. Die Bank hat der Stadt das Geld für das neue Schwimmbad geliehen.

b. Er hat einem Austauschstudenten sein WG-Zimmer bis Ende des Jahres für 300 Euro vermietet.

c. Die Verkäuferin hat mir eine Jacke für 180 Euro verkauft.

\footnotetext{
${ }^{7}$ Diese Konstruktionen werden hier als (syntaktisch-semantische) Strukturen (phrasal constructions) betrachtet, die lexikalisch unterschiedlich gefüllt werden. Als Strukturen lassen sie sich je nach Kontext und informationsstrukturellen Bedingungen unterschiedlich, d. h. in unterschiedlichen syntaktischen Realisierungsformen einsetzen. Valenzbedingte Variationen sowie Variationen, die sich aus informationsstrukturellen Faktoren ergeben, sind aus dieser Perspektive erst auf der diskursiven Ebene anzusiedeln und verlangen keine spezifischen Ableitungsregeln, wie sie bei lexical constructions (cf. Müller 2006) nötig wären.

8 Alle Beispiele unter (10) bis (12) wurden durch eine Google-Suche gefunden und dann zu einer besseren Veranschaulichung der Valenzverhältnisse vereinfacht.
} 
Diese Beispielsätze mit unterschiedlichen Basisverben instanziieren zunächst einmal die folgenden drei Szenarien ${ }^{9}$ :

- $\quad$ Transfer-Frame in (10)

- $\quad$ Sending-Frame in (11)

- Commercial-Frame in (12)

Diesen drei Frames gemeinsam ist eine stabile Struktur, die sich syntaktisch durch grammatikalische Funktionen und semantisch durch Rollen, wie in (13) dargestellt, schematisieren lässt:

$$
\begin{array}{llll}
\text { synt. } & \text { Subjekt } & \text { Dativ-Objekt } & \text { Akkusativ-Objekt } \\
\text { sem. } & \text { Agens } & \text { Benefaktiv } & \text { Affiziertes-Objekt }
\end{array}
$$

Auf dieser Basis kann die ditransitive Konstruktion, wie sie Goldberg (1995: 142) darstellt, als letzte Abstrahierungsstufe dieser Frames aufgefasst werden:

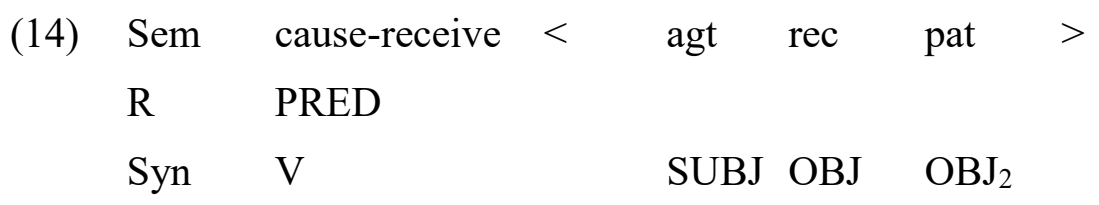

Dieses Schema veranschaulicht die Art und Weise, wie die syntaktische und die semantische Seite einer Konstruktion zusammen eine Ganzheit bilden: Auf der unteren Zeile werden die einzelnen syntaktischen Funktionen aufgelistet, welche die Konstruktion vorsieht (hier fürs Deutsche $^{10}$ : Subjekt, Dativ-Objekt, Akkusativ-Objekt), während die obere Zeile die so genannten Argumentrollen (argument roles bei Goldberg 1995: 43) beinhaltet, welche sehr allgemeinen semantischen Kategorien entsprechen (etwa nach den semantischen Rollen oder Tiefenkasus von Fillmore 1968, 1971, 1977). Die R-Zeile in der Mitte betrifft ihrerseits die Integration eines konkreten Verbes in dieses abstrakte Konstruktionsschema ${ }^{11}$, das eben durch Frame-Elemente definiert wird, z. B. donor, theme und recipient für ein Verb wie geben im TransferFrame. Boas (2011: 42) fasst diese Relation wie folgt zusammen, wobei er auch die Kompatibilitätsfrage zwischen den abstrakten Argumentrollen der Konstruktion und den Frame-Elementen stellt, die für unsere Problemstellung auch von besonderer Bedeutung ist (cf. Abschnitt 4):

Der vielleicht wichtigste Aspekt der Konstruktion ist die Zeile in der Mitte (R: instances, means), in die das Verb (PRED) unter bestimmten Umständen eingefügt werden kann. Wenn ein Verb mit einer Konstruktion fusioniert, dann geschieht das laut Goldberg dadurch, dass die Partizipantenrollen des Lexikoneintrags des Verbs in die mittlere Zeile (PRED) der Konstruktion eingefügt

\footnotetext{
${ }^{9}$ Die Bezeichnungen und Definitionen der einzelnen Frames sind der Datenbank des FrameNet-Projekts entnommen.

Transfer-Frame: „This frame involves a Donor transferring a Theme to a Recipient.” (FrameNet, https://framenet.icsi.berkeley.edu/fndrupal/index.php?q=frameIndex [15.01.2018])

Sending-Frame: „A Sender plans the Path (along with Source and Goal) of a Theme and places it in circumstances such that it travels along this Path under the power of some entity other than the Sender. This frame also has a Recipient distinct from the Goal, as both can be present.“ (FrameNet, https://framenet.icsi.berkeley.edu/ fndrupal/index.php?q=frameIndex [15.01.2018])

Commercial-Frame : „These are words that describe basic commercial transactions involving a Buyer and a Seller who exchange Money and Goods.“ (FrameNet, https://framenet.icsi.berkeley.edu/fndrupal/ index.php?q=frameIndex [15.01.2018])

${ }^{10}$ Goldberg arbeitet ja zum Englischen, wo die Frage der Deklination sich nicht stellt.

11 Goldberg (1995: 51): „The construction also specifies the way in which the verb is integrated into the construction - what type of relation R can be. Sometimes a specific relation, e. g. means or instances, replaces R in the diagrams $[\ldots] . “$
} 
Stufe 2: Agens und Patiens werden ausgedrückt, eine zusätzliche Instanz wird auch erwähnt, und zwar in der Rolle des Benefaktivs, während das Ziel in den prädikativen Ausdruck inkorporiert wird. Dies liegt an der besonderen semantischen Struktur von Funktionsverbgefügen, wo das so genannte Funktionsverb nur als Träger morpho-syntaktischer Kategorien fungiert und das Prädikat durch das Glied der Präpositionalgruppe ausgedrückt wird. Gerade durch die Inkorporierung in eine PP, deren Basis systematisch eine Bewegungspräposition ist, liegt es nahe, sie als Realisierung des Ziels aufzufassen, auch wenn sie nicht erfragbar ist (cf. Helbig 1979).

$\begin{array}{lllll}\text { (17) Wir } & \text { stellen } & \text { Ihnen } & \text { einen Platz } & \text { zur Verfügung. } \\ \text { SUBJ }_{\text {NOM }} & \text { VERB } & \text { OBJ }_{\text {DAT }} & \text { OBJ }_{\text {AKK }} & \text { PRÄP-P } \\ \text { AGENS } & \text { F-VERB } & \text { BENEF } & \text { AFFIZ.OBJ } & \text { HANDLUNGSPRÄD. (+ ZIEL) }\end{array}$

Stufe 4a: Hier haben wir es mit einer strikten Realisierung des Frames zu tun, allerdings mit einer größeren Auswahl an Verben, die sozusagen in ihrer wörtlichen Bedeutung verwendet werden:

\begin{tabular}{|c|c|c|c|}
\hline hat & das Auto & in den Graben & Gefahren \\
\hline $\mathrm{SUBJ}_{\mathrm{NOM}}$ & $\mathrm{OBJ}_{\mathrm{AKK}}$ & PRÄP-P & VERB \\
\hline AGENS & AFFIZ. OBJ & ZIEL & HANDLUNGSPRÄD. \\
\hline
\end{tabular}

$\mathrm{Ab}$ Stufe $4 \mathrm{~b}$ wird der Frame metaphorisch aktiviert, und zwar außerhalb des ursprünglichen Bewegungsbereichs: So wird die Konstruktion mit Verben möglich, die per se keine Bewegung ausdrücken. Es sind aber Einschränkungen zu verzeichnen, die unserer Hypothese zufolge auf die Frame-Elemente zurückgeführt werden können:

- $\quad$ nach wie vor sind Handlungsprädikate unentbehrlich, d. h. Prädikate, die das Vorhandensein eines Agens benötigen, welches ein Patiens sozusagen kontrolliert;

- $\quad$ die ,Bewegungs'-Komponente bleibt insofern erhalten, als der Übergang vom Zustand zum Zustand 2 metaphorisch als Bewegung konzeptualisiert wird, so zum Beispiel in der Struktur jmdn. in den Schlaf wiegen, wo das Patiens vom Wachzustand in den Schlafzustand überführt wird;

- das Ziel wird als Ort bzw. Container konzeptualisiert.

In diesem Zusammenhang kann es interessant sein, die existierenden Valenzbeschreibungen dieser Verben zu Rate zu ziehen ${ }^{14}$ : sie illustrieren nämlich die Parallele bzw. die Diskrepanz zwischen konventionalisiertem, d. h. in der Grammatik selbst verankertem Kausativitätsausdruck und auffälligen, d. h. durch lexikalische Füllung der abstrakten Konstruktion realisierten kausativen Äußerungen:

\footnotetext{
14 Zu diesem Zweck wurden zwei elektronische Ressourcen des Instituts für deutsche Sprache (IdS) bemüht. Einerseits das elektronische Valenzwörterbuch valbu, das für eine Auswahl an deutschen Verben, die sich an die Wortschatzliste des ,Zertifikats Deutsch“ anlehnt, eine detaillierte Beschreibung ihrer syntaktischen und semantischen Umgebungen anbietet und sie durch Verwendungsbeispiele veranschaulicht. Andererseits das Online-Wörterbuch zur deutschen Gegenwartssprache des IdS, das den Wortschatz der deutschen Sprache anhand von aktuellen Sprachdaten dokumentiert, erklärt und wissenschaftlich kommentiert. Beide Ressourcen sind online frei zugänglich unter: http://hypermedia.ids-mannheim.de/evalbu/index.html und www.owid.de/wb/elexiko/start.html [15.01.2018].
} 
- legen: drei Valenzstellen sind in e-valbu ${ }^{15}$ erwähnt: ,jemand ${ }_{1}$ legt jemanden $2 /$ etwas 2 irgendwohin $_{3}$ im Sinne von,,jemand bringt jemanden/etwas irgendwohin und versetzt ihn/sie/es auf einer Fläche in eine waagerechte, liegende Position" mit drei Belegungsre-

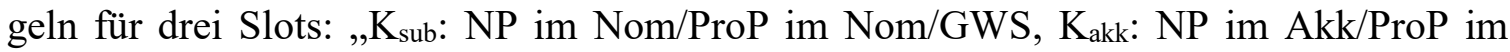
Akk/GWS, $\mathrm{K}_{\mathrm{adv}}$ : Ort (direktivisch)“.

- fällen: ist nicht in e-valbu vorhanden. Die in elexiko ${ }^{16}$ in der Rubrik „Kollokationen“ angeführten Informationen ermöglichen es aber, das Valenzprogramm zu rekonstruieren, und zwar mit den Slots „Wer fällt etwas?“ und „Was wird gefällt?“. Syntaktisch gesehen hat man es mit „, $\mathrm{K}_{\text {sub: }}$ NP im Nom/ProP im Nom, $\mathrm{K}_{\text {akk }}$ : NP im Akk/Prop im Akk“ zu tun, wobei semantisch die Realisierung von $\mathrm{K}_{\mathrm{akk}}$ auf die Klasse ,Baum' begrenzt ist.

Die Belegsätze (15) und (16) entsprechen ganz genau diesen beiden Schemata.

- $\quad$ stellen: das Verb in der hiesigen Verwendung wird in e-valbu separat behandelt, und zwar als Funktionsverb innerhalb eines Funktionsverbgefüges. Dies liefert, wenn nötig, einen zusätzlichen Beweis für die oben angesprochene Integration dieser Strukturen in die Phraseologie.

- fahren: e-valbu sieht auch ein 3-stelliges Programm vor: ,jemand ${ }_{1}$ fährt etwas 2 irgendwo-

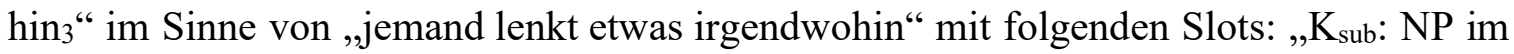
Nom/ProP im Nom/GWS, Kakk: NP im Akk/ProP im Akk/GWS, Kadv: Ort (direktivisch)“

Hier auch realisiert der Belegsatz ganz genau dieses Schema. Ab Stufe $4 \mathrm{~b}$ hat man es aber mit Realisierungsformen zu tun, die zumindest im syntaktischen Valenzprogramm der Verben nicht vorgesehen sind, weshalb sich der Umweg über die zugrunde liegenden Frames als notwendig erweist:

- wiegen: in diesem Sinne in e-valbu nicht vorhanden

- singen: in dieser Konstruktion in e-valbu deutlich als Irregularität charakterisiert: „singen wird auch in dem Ausdruck jemanden in den Schlaf singen verwendet i. S. v. 'so lange singen, bis jemand schläft'“".

- tanzen: reflexive Konstruktion in e-valbu nicht vorhanden

- $\quad$ klingeln: in dieser Konstruktion in e-valbu deutlich als Irregularität charakterisiert; ,klingeln wird auch in dem Ausdruck jemanden aus dem Bett/aus dem Schlaf klingeln i. S. v. ,so lange die Klingel betätigen oder das Telefon läuten lassen, bis jemand aufsteht/aufwacht und aufmacht oder ans Telefon geht" verwendet".

Auf dieser Basis lässt sich die Gesamtbedeutung solcher Konstruktionen durch zwei Merkmale charakterisieren: Sie sind zunächst einmal nicht streng kompositionell, sie sind dann unabhängig von der Verbbedeutung, und gerade hier zeigt sich die Relevanz des konstruktivistischen Ansatzes: Während die Definition der Frames in (10), (11) und (12) die Bedeutung des Verbes und einen gewissen Anteil an Weltwissen voraussetzt, ist es hier nicht mehr der Fall; die Gesamtbedeutung kann letzten Endes nur auf die Struktur des Ganzen zurückgeführt werden: Erst

\footnotetext{
15 Cf. http://hypermedia.ids-mannheim.de/evalbu/index.html [15.10.2018].

16 Cf. www.owid.de/artikel/124682/abholzen?module=elex_b [15.01.2018].
} 
das Vorhandensein aller hier besprochenen Frame-Elemente ermöglicht die kausative Dekodierung.

\section{$4 \quad$ Zur phraseologischen Relevanz solcher Konstruktionen}

Kommen wir nun auf die anfangs gestellte Frage zurück: Sind caused-motion-Konstruktionen als Teil des phraseologischen Systems des Deutschen zu betrachten bzw. wo gibt es Berührungspunkte mit phraseologischen Einheiten?

Im Folgenden werden wir - über die üblichen Definitionskriterien für Phraseme hinaus - das Kontinuum der caused-motion-Konstruktionen noch einmal unter die semantische Lupe nehmen und uns in erster Linie mit dem Kriterium der Metaphorizität befassen. Uns geht es dabei um zweierlei: einerseits um die Möglichkeit, bestimmte Verben unabhängig von ihrem Valenzrahmen in Konstruktionen mit einer Akkusativergänzung als affizierte Größe und einer Präpositionalphrase als Zielrichtung oder Herkunft zu verwenden, und andererseits um die Interpretation solcher Konstruktionen.

\subsection{Kriterien}

Die drei traditionellen Definitionskriterien für Phraseme (nämlich Polylexikalität, Fixiertheit und Figuriertheit, cf. Abschnitt 1) sind bei den caused-motion-Konstruktionen recht unterschiedlich realisiert. Während sie alle polylexikalisch sind, werden die beiden anderen Kriterien der Festigkeit und der Figuriertheit nur bei einem Teil dieser Konstruktionen erfüllt. Interessanterweise stellt man fest, dass die Festigkeit mit den syntaktischen (d. h. valenzbezogenen) Abweichungsmöglichkeiten zusammenhängt und dass solche syntaktischen Abweichungen einerseits einige feste lexikalische Verbindungen aufweisen ${ }^{17}$ und andererseits oft Ausdrücke mit einer metaphorischen Komponente betreffen. Auf diese beiden Aspekte wollen wir hier kurz eingehen.

\subsection{Syntaktisch-lexikalische Festigkeit}

Abgesehen von den Verben, die primär eine caused-motion-Handlung ausdrücken, haben wir es in dem vorgeschlagenen Kontinuum dann mit syntaktischen Gefügen zu tun, die sich durch bestimmte syntaktisch-lexikalische Regularitäten oder gar lexikalische Füllungen auszeichnen. Neben den FVG, die relativ fest und auf wenige Verben beschränkt sind, sind es einerseits die reflexiven Konstruktionen und andererseits die anderen, durch ihre abweichende Transitivität auffälligen Konstruktionen. Bei ersteren besetzt das Reflexivpronomen die Stelle einer Akkusativergänzung, die sonst beim freien Gebrauch des Verbs nicht vorgesehen ist (zumindest (rollen)semantisch nicht als solche):

(19) sich in das Herz des Publikums tanzen/singen/spielen

Bei letzteren haben wir es mit einer NP als Akkusativergänzung zu tun, die ebenfalls beim freien Gebrauch des Verbs entweder (rollen)semantisch anders besetzt werden muss (20) oder nicht vorgesehen ist (21):

${ }^{17}$ Cf. Sinclair (1991: 65): „It seems that there is a strong tendency for sense and syntax to be associated“. 
(20) jemanden in den Schlaf singen

(21) jemanden aus dem Bett klingeln

In beiden Fällen sind es hier außerdem Verben, die in ihrem freien Gebrauch bzw. in anderen nicht metaphorisch motivierten Konstruktionen auf Handlungen oder Aktivitäten verweisen, die an sich keine Ortsveränderung bewirken oder bewirken müssen. Warum sie aber als Instanzen solcher Konstruktionen gebraucht werden können und beim Ausdruck einer Ortsveränderung mitwirken und vor allem warum nicht jedes beliebige Verb hier - bei aller Produktivität der Konstruktionen - ,toleriert' wird, haben wir weiter oben zu erklären versucht, und zwar zugleich durch die als Bewegung konzeptualisierte Zustandsänderung und durch die Ort-/Container-Metapher. Wir werden im nächsten Abschnitt auf die Semantik der betroffenen Verben näher eingehen. Ein aus unserer Sicht wichtiger Faktor ist die Metaphorizität der Präpositionalgruppe bzw. des ,Ortes' (Ziel oder Pfad); der metaphorische Mechanismus ist es, der einerseits zur Erweiterung des Kontinuums sowie zur Variation bei der lexikalischen Füllung der Stellen beiträgt. So enthält die Konstruktion in (20) eine durch die PP ausgedrückte direktionale Ortsveränderung, bei der der ZIEL-Slot durch einen Zustand (Schlaf) instanziert wird. Dadurch rückt sie semantisch in die Nähe der Resultativ-Konstruktionen, die ja auf einen durch ein Adjektiv ausgedrückten Zustand hinweisen (cf. sein Hemd nass schwitzen), jedoch bleibt sie auf der Form-Ebene (durch die Präpositionalphrase, cf. in den Schlaf wiegen) eindeutig dem Bereich der caused-motion-Konstruktionen sehr nah.

\subsection{Interpretation}

Nach Goldberg (1995: 81-84) sorgt unter den verschiedenen Vererbungsbeziehungen die metaphorische Erweiterung dafür, dass sich solche Konstruktionen wie unter (19) und (20) im Sprachgebrauch etablieren und ohne weiteres interpretieren lassen. Verschiedene Autoren haben schon darauf hingewiesen, dass Metaphern als kognitiver Mechanismus Realisierungen von Konstruktionen ermöglichen, die einen syntaktisch abweichenden Charakter haben, jedoch interpretierbar sind (cf. zuletzt Baicchi 2011; del Campo 2013; Peña 2009; Ziem 2015; im Rahmen des Lexical Constructional Models: Ruiz de Mendoza Ibáñez/Mairal Usón 2007; Ruiz de Mendoza Ibáñez/Luzondo-Oyón 2014). Die Interpretierbarkeit stützt sich einerseits auf semantische Projektionen (zu den sog. ,priming effects“ cf. u. a. Ellis et al. 2014: 57f.), andererseits aber auch auf den Zugriff auf kognitiv zugängliche Szenen, die bestimmte lexikalische Füllungen erlauben oder umgekehrt blockieren, weshalb Frame-Semantik in ihrer ganzen Brandbreite ${ }^{18}$ hier auch bemüht werden kann bzw. soll.

Semantische Vererbungsbeziehungen könnten für eine begrenzte Erweiterung der Konstruktionen auf lexikalischer Ebene sorgen, jedoch erklärt dieses Prinzip nicht, warum bestimmte Verben möglich sind, deren Valenz ihren Gebrauch in caused-motion-Konstruktionen eigentlich unmöglich machen sollte. ${ }^{19}$ Der Vorschlag von Boas (2011), lexikalisch spezifizierte Minikon-

\footnotetext{
${ }^{18}$ Goldberg ist ja oft vorgeworfen worden, in ihrer Konstruktionsgrammatik eine reduzierte Auffassung der Frame-Semantik zu haben (cf. Boas 2011: 49f.).

${ }^{19}$ Cf. auch del Campo (2013: 90): "But constructionist views have not specified what it is that licenses or blocks the integration of predicates into syntactic structures."
}

ISSN 1615-3014 
struktionen einzuführen, um schon auf der Ebene der Verbbedeutung einen relativ konventionalisierten Frame einzusetzen, in dem bestimmte Rollen vorhanden sind, wäre hier eine semantische Erklärung für das Vorhandensein von Slots wie Herkunftsort oder Pfad, die - von Verb aus gesehen - rein syntaktisch als unzulässig betrachtet werden. So erklärt sich der Gebrauch von klingeln in caused-motion-Konstruktionen, da die Handlung meistens ausgeführt wird, damit jemand die Tür aufmacht, was eine Bewegung durch einen Raum impliziert. Warum ein solcher Frame für das Verb klingeln gilt, aber nicht für klopfen, das eine ähnliche Handlung mit einem ähnlichen Ziel ausdrückt und eine solche Konstruktion nicht lizenziert, bleibt jedoch unerklärt. Es hängt womöglich mit der Intensität des Geräuschs zusammen sowie mit dem ganzen Wissen zur prototypischen außersprachlichen Ausgangsszene: aufstehen, (sich ankleiden), zur Tür gehen, die Tür aufmachen, usw. Dieser Erklärungsansatz plädiert für den Umweg über Frame-Semantik und, wie schon lange von Busse (1997) explizit formuliert, gegen die oft postulierte Trennung zwischen lexikalischem und nicht-lexikalischem Wissen:

Der größte Teil des verstehensrelevanten Wissens jedoch - vor allem das, was man im üblichen Verständnis zur Semantik rechnet - ist in dem Sinne nicht allein sprachlich, dass es nicht mit ausschließlich linguistischen Mitteln beschrieben werden kann, sondern mit Mitteln, welche allgemeine enzyklopädische Informationen und Beschreibungsverfahren zu hilfe nehmen, beschrieben werden muss.

(Busse 1997: 29)

Seinem Vorschlag, von einem, ,verstehensrelevanten Wissen“ zu sprechen, um eben diese Trennung aufzuheben, ist hier um so mehr zu folgen, als dass wie vorher erwähnt die Erklärung für die Zulassung solcher Konstruktionen beim Rezipienten eindeutig in Richtung der kognitiven Rekonstruktionsmöglichkeiten zu suchen ist.

In Konstruktionen vom Typ 4 (mit Reflexivpronomen) spielen konzeptuelle Metaphern eine entscheidende Rolle bei der Interpretation der Entität, die in der PP enthalten ist, als Ziel oder Herkunftsort/Pfad. So die oben angeführten Beispiele mit Herz als Behälter für Gefühle und hier für große Sympathie oder gar Liebe. Der Konventionalisierungsgrad oder zumindest die Geläufigkeit der jeweiligen konzeptuellen Metapher sorgt für die Interpretierbarkeit der Konstruktion: Insofern unterliegt die PP gewissen kognitiven Zwängen. Auf der anderen Seite aber ist die Realisierungsform des Prädikats/Verbs freier - vorausgesetzt, die Handlung hat (mit ihrem Ziel) für die Sprecher eine gewisse/hinreichende Evidenz (cf. „patterns of experience“, Goldberg 1998 ${ }^{20}$. Schaut man sich Korpusbelege $a^{21}$, so stellt man fest, dass es sich hier vornehmlich - in Verbindung mit ,ins Herz / in die Herzen + NPGen.“ - um künstlerische (auch sportliche) Leistungen handelt, bei denen ein Rezipient (Zuschauer oder Zuhörer) und ein Ziel (Gefallen) mitgedacht ist. Eine entsprechende lexikalische bzw. lexikografische Erfassung dieser Verben und der entsprechenden verstehensrelevanten Komponenten (i. e. Frames) könnte ihren Gebrauch in solchen Konstruktionen berücksichtigen.

\footnotetext{
${ }^{20}$ Im Englischen kommen Metaphorisierungen von Verben in solchen Konstruktionen häufiger vor; cf. She showed me out of the problem, zitiert von Baicchi (2011: 159-166).

${ }^{21}$ Eine Cosmas-Recherche im DeReko (IDS/ Mannheim) hat für die PP in die Herzen die meisten Kookkurrenzen mit dem Verb spielen gezeigt (1968 Treffer); andere häufige Kookkurrenzpartner sind singen (1692 Treffer) und tanzen (675 Treffer).
} 
Durch diese zusätzliche Einschränkung pragmatischer Art erklärt sich der hohe Grad an Motiviertheit der metaphorischen caused-motion-Konstruktionen. Bei den Konstruktionen vom letzten Typ (jmdn. in den Schlaf singen/jmdn. aus dem Bett klingeln) wird die Motiviertheit noch deutlicher; ob der Zustand des Schlafes als erreichten Ort gesehen wird oder der Ort ,Bett' für den Zustand der betroffenen Person verstanden wird, in beiden Fällen stützt sich die Konstruktion auf erfahrungsgemäß trivialen Handlungen. Auch wenn solche Fälle an der Grenze zur Resultativität liegen, gehen wir hier auf das Problem der Unterscheidung bzw. der Beziehung22 zwischen resultativen und caused-motion-Konstruktionen nicht ein (cf. u. a. Goldberg/Jackendoff 2004; Ettlinger 2005 und 2008) ${ }^{23}$.

Geht man also von einem möglichen Kontinuum innerhalb des Konstruktionssystems einer Sprache aus, dann befinden sich die caused-motion-Konstruktionen auf einer Skala zwischen leeren Argument-Strukturen, die relativ frei gefüllt werden können, am einen Ende, und lexikalisch fixierten Schemata am anderen Ende und gehören zu den Strukturen, die zwar lexikalisch relativ unbestimmt sind, aber kognitiv starken Einschränkungen unterliegen. Gerade da stehen sie an der phraseologischen (durchlässigen) Grenze.

\section{$5 \quad$ Fazit}

Die hier angesprochenen Aspekte sollten Argumente für oder gegen eine Integration der caused-motion-Konstruktionen in die phraseologischen Einheiten der Sprache liefern. Der Mangel an lexikalischer Festigkeit spricht eher gegen eine solche Integration. Dies entspricht z. B. dem Standpunkt von Dobrovol'skij (2011: 112), der für die Aufnahme von Einheiten ,mit maximaler lexikalischer Füllung" plädiert und Konstruktionen wie die Phrasem-Konstruktionen (sog. „Phraseoschablonen“) als am wenigsten repräsentativ betrachtet ${ }^{24}$.

Dennoch weisen manche caused-motion-Konstruktionen Züge auf, durch die sie wiederum in die Nähe der Phraseologie rücken: syntaktische Abweichung bei einer gewissen Produktivität; Einschränkungen im Gebrauch, die mit den sprachsystembedingten Einschränkungen nicht korrelieren; metaphorische Komponente. Hinzu kommt wahrscheinlich die parallele Speicherung als Ganzes im mentalen Lexikon - eine Hypothese die noch zu verifizieren ist, etwa durch Daten aus dem (Früh-)Spracherwerb (Ochsenbauer/Hickmann 2010).

Eine sehr weit gefasste Definition, wie sie von Gries (2008) gegeben wird, könnte einen Teil unserer Konstruktionen in den Bereich der Phraseologie retten:

In sum, a phraseologism is defined as the cooccurrence of a form or a lemma of a lexical item and one or more additional linguistic elements of various kinds which functions as one semantic unit in a clause or sentence and whose frequency of co-occurrence is larger than expected on the basis of chance.

(Gries 2008: 6)

\footnotetext{
22 Goldberg (1995: 81-84) postuliert eine metaphorische Relation zwischen den beiden Typen von Konstruktionen.

23 Zur Diskussion über die Abgrenzung zwischen caused-motion-Konstruktionen bzw. caused-motion-patterns und resultativen Konstruktionen verweisen wir auf Kay (2005) und Boas (2011).

${ }^{24}$ Cf. Stumpf (2015) für eine ähnliche Diskussion des phraseologischen Status der , $\mathrm{X}_{\mathrm{NOM}}$ können $\mathrm{Y}_{\mathrm{AKK}}{ }^{6}-$ und ,können $\mathrm{X}_{\mathrm{NOM}} \mathrm{Y}_{\mathrm{AKK}}$ ?'-Konstruktionen.
} 
Um auf die eingangs gestellte Frage zurückzukommen, kann provisorisch festgehalten werden, dass konstruktionsgrammatische Ansätze - über die Frage der Zugehörigkeit bestimmter Einheiten zur Phraseologie oder nicht - die Notwendigkeit deutlich in den Vordergrund rücken, Fixiertheit und Figuriertheit zu hinterfragen und neu zu beleuchten. Die hier vorgeschlagenen Erklärungen für eine semantisch-syntaktische Dekodierung von caused-motion-Konstruktionen im Deutschen betonen ja deren idiosynkratrische Komponenten sowie die starken syntaktischen Irregularitäten und semantischen Restriktionen, denen sie unterliegen, was ihre Behandlung in einer Grauzone zwischen Phraseologie und constructicon als Teil des mentalen Lexikons auch rechtfertigen kann.

\section{Literatur}

Baicchi, Annalisa (2011): "Metaphoric motivation in grammatical structure. The caused-motion construction from the perspective of the Lexical-Constructional-Model". In: Panther, Klaus-Uwe/Radden, Günter (eds.): Motivation in Grammar and the Lexicon. Amsterdam/Philadelphia, Benjamins: 149-169. (= Human Cognitive Processing 27).

Boas, Hans (2003): A constructional Approach to Resultatives. Standford: CSLI Publications.

Boas, Hans (2008): "Resolving form-meaning discrepancies in Construction Grammar". In: Leino, Jakko (ed.): Constructional Reorganization. Amsterdam/Philadelphia, Benjamins: 11-36. (= Construcional Approaches to Language 5).

Boas, Hans (2011): „Zum Abstraktionsgrad von Resultativkonstruktionen“. In: Engelberg, Stefan/Proost, Kristel/Holler, Anke (eds.): Sprachliches Wissen zwischen Lexikon und Grammatik, Berlin/New York, Mouton de Gruyter: 37-69. (= IdS-Jahrbuch 2010).

Burger, Harald (1973): Idiomatik des Deutschen. Tübingen: Niemeyer. (= Germanistische Arbeitshefte 16).

Burger, Harald (2010): Phraseologie: eine Einführung am Beispiel des Deutschen. 4. neu bearbeitete Auflage. Berlin: Erich Schmidt. (= Grundlagen der Germanistik 36).

Burger, Harald/Häcki Buhofer, Annelies/Sialm, Ambros (1982): Handbuch der Phraseologie. Berlin/New York: de Gruyter.

Burger, Harald/Dobrovol'skij, Dmitrij/Kühn, Peter/Norrick, Neal (eds.) (2007): Phraseologie: ein internationales Handbuch zeitgenössischer Forschung. Berlin/New York: de Gruyter. $(=$ HSK 28.1-28.2).

Busse, Dietrich (1997): „Semantisches Wissen und sprachliche Information. Zur Abgrenzung und Typologie von Faktoren des Sprachverstehens“. In: Pohl, Inge (ed.): Methodologische Aspekte der Semantikforschung. Frankfurt a. M., Peter Lang: 13-34. (= Sprache. System und Tätigkeit 22).

Dalmas, Martine (2006): „Wie plattgesessene Currywürste und nassgepisste Klodeckel entstehen. Wahlverwandtschaften der 3. Art.“ In: Marillier, Jean-François/Dalmas, Martine/Behr, Irmtraud (eds.): Text und Sinn. Studien zur Textsyntax und Deixis im Deutschen und im Französischen. Tübingen, Stauffenburg: 291-304. (= Eurogermanistik 23).

del Campo Martinez, Nuria (2013): "The metaphoric motivation of the caused-motion construction. A case study of perception“. International Journal of English Studies 13/1: 89-110.

Dixon, Robert (2000): “A typology of causatives: form, syntax and meaning”. In: Dixon, Robert/Aikhenvald, Alexandra (eds.): Changing valency Case studies in transitivity. Cambridge, Cambridge University Press: 30-83. 
Dobrovol'skij, Dmitrij (2011): „Phraseologie und Konstruktionsgrammatik“. In: Lasch, Alexander/Ziem, Alexander (eds.): Konstruktionsgrammatik III. Aktuelle Fragen und Lösungsansätze. Tübingen, Stauffenburg: 111-130. (=Stauffenburg Linguistik 58).

Dobrovol'skij, Dmitrij/Piirainen, Elisabeth (2009): Zur Theorie der Phraseologie. Kognitive und kulturelle Aspekte. Tübingen: Stauffenburg. (= Stauffenburg Linguistik 49).

Ellis, Nick/Brook O’Donnell, Matthew/Römer, Ute (2014): “The processing of verb-argument constructions is sensitive to form, function, frequency, contigency and prototypicality". Cognitive Linguistics 25/1: 55-98.

Ettlinger, Marc (2005): Constructional Compositionality and the English resultative. Ms. 41st Meeting of the Chicago Linguistics Society. Chicago: Chicago Linguistics Society.

Ettlinger, Marc (2008): "The Syntactic Behavior of the Resultative: Evidence for a Constructional Approach". Proceedings of 41st Annual Meeting of the Chicago Linguistics Society. Vol. 2. Chicago, Chicago Linguistics Society: 145-160.

Fillmore, Charles (1968): "The Case for the Case". In: Bach, Emmon/Harms Robert (eds.): Universals in Linguistic Theory. New York, Holt, Rinehart and Winston: 1-88.

Fillmore, Charles (1971): "Some Problems for Case Grammar". In: O’Brian, Richard (ed.): Report on the Twenty-Second Annual Round Table Meeting on Languages and Linguistics. Washington, Georgetown University Press: 35-56.

Fillmore, Charles (1977): “The Case for Case reopened”. In: Cole, Peter/Sadock, Jerrold (eds.): Grammatical Relations. New York, Academic Press: 59-82 (= Syntax and Semantics 8).

Fleischer, Wolfgang (1997): Phraseologie der deutschen Gegenwartssprache. 2. durchgesehene und ergänzte Auflage. Tübingen: Niemeyer.

Fried, Mirjam (2009): "Representing contextual factors in language change: between frames and constructions". In: Bergs, Alexander/Diewald, Gabriele (eds.): Context and constructions. Amsterdam/Philadelphia, Benjamins: 63-94. (= Constructional Approaches to Language 9).

Goldberg, Adele (1995): Constructions: A Construction Grammar Approach to Argument Structure. Chicago/London: The University of Chicago Press.

Goldberg, Adele (1998): "Patterns of Experience in Patterns of Language". In: Tomasello, Michael (ed.): The New Psychology of Language. Mahwah u. a., Lawrence Erlbaum Publications: 203-219.

Goldberg, Adele (2006): Constructions at Work: The Nature of Generalization in Language. Oxford: Oxford University Press.

Goldberg, Adele/Jackendoff, Ray (2004): "The English resultative as a family of constructions". Language 80: 532-569.

Granger, Sylviane/Meunier, Fanny (eds.) (2008): Phraseology: an interdisciplinary perspective. Amsterdam/Philadephia: Benjamins.

Gries, Stefan (2008): "Phraseology and linguistic theory. A brief survey". In: Granger, Sylviane/Meunier, Fanny (eds.): Phraseology: an interdisciplinary Perspective. Amsterdam, Benjamins: 3-25.

Gries, Stefan/Stefanowitsch, Anatol (2004): "Co-varying collexemes in the into-causative“. In: Achard, Michel/Kemmer, Suzanne (eds.): Language, Culture, and Mind. Stanford, CSLI: 225-36. 
Hampe, Beate (2011): "Metaphor, constructional ambiguity and the causative resultatives". In: Handl, Sandra/Schmid, Hans-Jörg (eds.): Windows to the Mind. Berlin/New York, Mouton de Gruyter: 185-215. (= Cognitive Linguistics Research 48).

Helbig, Gerhard (1979): „Probleme der Beschreibung von Funktionsverbgefügen im Deutschen“. Deutsch als Fremdsprache 16: 273-285.

Heringer, Hans-Jürgen (1968): Die Opposition von ,kommen' und , bringen' als Funktionsverben: Untersuchungen zur grammatischen Wertigkeit und Aktionsart. Düsseldorf: Schwann. (= Sprache der Gegenwart 3).

Kamber, Alain (2008): Funktionsverbgefüge - empirisch. Eine korpusbasierte Untersuchung zu den nominalen Prädikaten des Deutschen. Tübingen: Max Niemeyer. (= Reihe Germanistische Linguistik 281).

Kamber, Alain (2006): „Funktionsverbgefüge - empirisch (am Beispiel von kommen)“. Linguistik online 28.

Kay, Paul (2005): “Argument structure constructions and the argument adjunct distinction”. In: Fried, Mirjam/Boas, Hans (eds.): Grammatical Constructions: Back to the Roots. Amsterdam, Benjamins: 71-98. (= Constructional Approaches to Language 4).

Müller, Stefan (2002): Complex Predicates: Verbal Complexes, Resultative Constructions, and Particle Verbs in German. Standford: CSLI Publications.

Müller, Stefan (2006): „Phrasal oder Lexical Constructions?“. Language 82(4): 850-883.

Nemoto, Noriko (1998): "On the Polysemy of Ditransitive save: The Role of Frame Semantics in Construction Grammar". English Linguistics 15: 219-242.

Ochsenbauer, Anne-Katharina/ Hickmann, Maya (2010): “Children's verbalizations of motion events in German". Cognitive Linguistics 21/2: 217-238.

Peña Cervel, Sandra (2009): "Constraints on subsumption in the caused-motion construction". Language Sciences 31: 740-765.

Perek, Florent/Hilpert, Martin (2014): “Constructional tolerance. Cross-linguistic differences in the acceptability of non conventional uses of constructions". Constructions and frames 6/2: 266-304.

Ruiz de Mendoza Ibáñez, Franciso/Mairal Usón, Ricardo (2007): "Levels of semantic representation: where lexicon and grammar meet”. Interlinguistica 17: 28-47.

Ruiz de Mendoza Ibáñez, Franciso/Luzondo-Oyón, Alba (2014): "Figurative and non-figurative motion in the expression of result in English". Language and Cognition 41: 1-27.

Sinclair, John (1991): Corpus, Concordance, Collocation. Oxford: Oxford University Press.

Stumpf, Sören (2015): „,Kann Joggi Weltmeister?‘ Phraseologische und konstruktionsgrammatische Überlegungen zu einer aus (laien-)sprachkritischer Sicht ,agrammatischen' Konstruktion“. Aptum. Zeitschrift für Sprachkritik und Sprachkultur 11/1: 1-20.

van Pottelberge, Jeroen (2007): „Funktionsverbgefüge und verwandte Erscheinungen“. In: Burger, Harald/Dobrovol'skij, Dmitrij/Kühn, Peter/Norrick, Neal (eds.): Phraseologie: ein internationales Handbuch zeitgenössischer Forschung. Bd 1. Berlin/New York: de Gruyter: 436-444 (= HSK 28.1).

van Pottelberge, Jeroen (2001): Verbonominale Konstruktionen, Funktionsverbgefüge: Vom Sinn und Unsinn eines Untersuchungsgegenstandes. Heidelberg: Winter. (= Germanistische Bibliothek 12).

Wray, Alison (2002): Formulaic Language and the Lexicon. Cambridge: Cambridge University Press. 
Wray, Alison (2008): Formulaic Language: Pushing the Boundaries. Oxford: Oxford University Press.

Ziem, Alexander (2014): „Von der Kasusgrammatik zum FrameNet: Frames, Konstruktionen und die Idee eines Konstruktikons“. In: Ziem, Alexander/Lasch, Alexander (eds.): Grammatik als Netzwerk von Konstruktionen? Sprachwissen im Fokus in der Konstruktionsgrammatik. Berlin/New York, de Gruyter: 263-290 (= Sprache und Wissen 15).

Ziem, Alexander (2015): ,(Konzeptuelle) Metaphern in der Kognitiven Konstruktionsgrammatik“. In: Spieß, Constanze/Köpcke, Klaus-Michael (eds.): Metapher und Metonymie. Theoretische, methodische und empirische Zugänge. Berlin/München/Boston, de Gruyter: 5177. (=Empirische Linguistik 1): 51-80. 